\title{
Isokinetic evaluation of muscular function in patients with chronic pain and fatigability
}

\author{
D. Maquet ${ }^{\mathrm{a}}$, J.L. Croisier ${ }^{\mathrm{a}}$, M. Moutschen ${ }^{\mathrm{b}}$ and J.M. Crielaard ${ }^{\mathrm{a}}$ \\ ${ }^{\mathrm{a}}$ Department of Physical Medicine and Rehabilitation, University of Liege, Belgium \\ ${ }^{\mathrm{b}}$ Department of Internal Medicine (Immunology), University of Liege, Belgium
}

Fatigue, widespread muscle pain and weakness represent characteristics of patients with fibromyalgia (FM). The objectives of the study were to measure muscle performances in women with FM and to compare the results with normal values as well as results of patients suffering from chronic fatigue syndrome (SFC).

Subjects were 41 women with FM $(41 \pm 10$ years old), 11 women with SFC (45 \pm 13 years old) and 124 healthy female controls physically inactive (38 \pm 13 years old). All subjects sustained the following measurements:

- maximal concentric isokinetic muscle strength of the knee extensors and flexors in the dominant limb at $60 \mathrm{deg} / \mathrm{sec}$ angular velocity. Absolute (N.m) and normalized (N.m/kg of body weight) peak torques (PT) were analyzed.

- muscle fatigue resistance through 30 maximal concentric isokinetic contractions of the knee flexors and extensors at $180 \mathrm{deg} / \mathrm{sec}$. Absolute (J) and normalized (J/kg of body weight) bilateral cumulative works developed by knee flexors and extensors were analyzed.

- static endurance by means of posture maintenance. We determined how long the participant could maintain the given posture (sec).

- isometric grip strength $(\mathrm{kg})$ on a Colin dynamometer.
Pain resulting from fatigue protocol was evaluated using a visual analogue scale (VAS in arbitrary units, a.u.; 0 represents "no pain" and 10 represents "maximal pain").

All muscle variables were significantly decreased in the FM patients as compared to the controls but also more surprisingly to the SFC patients. In contrast, muscle performances in SFC patients were not systematically reduced as compared to the control values. VAS pain score after fatigue protocol were higher in the FM and SFC groups.

Although these pathological states are often considered "overlapping" syndromes, the existence of specific muscle function perturbation in patients with FM suggest a possible particular alteration in these subjects. The impaired muscle performances noted in FM patients may be attributed to lower muscle levels of phosphagens, enzymatic abnormalities and thickening of the capillary endothelium [1].

\section{Reference}

[1] J.H. Park et al., Use of P-31 magnetic resonance spectroscopy to detect metabolic abnormalities in muscles of patients with fibromyalgia, Arthritis Rheum 41 (1998), 406-413. 
Table 1

Comparison of isokinetic, static endurance and grip strength parameters in FM patients, SFC patients and healthy control women

\begin{tabular}{lrrr}
\hline & Controls & SFC patients & FM patients \\
\hline Normalized PT EXT 60 $/ \mathrm{s}(\mathrm{N} . \mathrm{m} / \mathrm{kg})$ & $2.1(0.4)^{\mathrm{a}}$ & $1.9(0.4)^{\mathrm{a}}$ & $1.5(0.4)^{\mathrm{b}}$ \\
Normalized PT FLE 60\% $/ \mathrm{s}(\mathrm{N} . \mathrm{m} / \mathrm{kg})$ & $1.17(0.25)^{\mathrm{a}}$ & $0.9(0.15)^{\mathrm{c}}$ & $0.72(0.23)^{\mathrm{b}}$ \\
Cumulative work $(\mathrm{J})$ & $6644(1527)^{\mathrm{a}}$ & $5729(1097)^{\mathrm{c}}$ & $4093(1405)^{\mathrm{b}}$ \\
Normalized cumulative work $(\mathrm{J} / \mathrm{kg})$ & $115(24)^{\mathrm{a}}$ & $91(18)^{\mathrm{c}}$ & $64(24)^{\mathrm{b}}$ \\
Upper limb abduction (sec) & $299(11)^{\mathrm{a}}$ & $343(296)^{\mathrm{a}}$ & $132(84)^{\mathrm{b}}$ \\
Dominant hip flexion $(\mathrm{sec})$ & $260(174)^{\mathrm{a}}$ & $114(29)^{\mathrm{c}}$ & $63(49)^{\mathrm{b}}$ \\
Grip strength (kg) & $28(6)^{\mathrm{a}}$ & $26(6)^{\mathrm{a}}$ & $21(6)^{\mathrm{b}}$ \\
\hline
\end{tabular}

Difference in letters represents statistically significant difference.

Table 2

Comparison of VAS pain scores in FM patients, SFC patients and healthy control women

\begin{tabular}{cccc}
\hline & Controls & SFC patients & FM patients \\
\hline VAS score (a.u.) & $3(2)^{\mathrm{a}}$ & $6(3)^{\mathrm{b}}$ & $6(2)^{\mathrm{b}}$ \\
\hline
\end{tabular}

Difference in letters represents statistically significant difference. 